\title{
Clinical Significance of Epidurography Contrast Patterns after Adhesiolysis during Lumbar Percutaneous Epidural Neuroplasty
}

\author{
Sang-Hyuk Park, ${ }^{1}$ Gyu Yeul Ji $\mathbb{D}^{2},{ }^{2}$ Pyung Goo Cho, ${ }^{3}$ Dong Ah Shin $\mathbb{D}^{,},{ }^{2}$ Young Sul Yoon, \\ Keung Nyun Kim, ${ }^{2}$ and Chang Hyun $\mathrm{Oh}^{4}$ \\ ${ }^{1}$ Department of Neurosurgery, Yonsei Barun Hospital, Seoul, Republic of Korea \\ ${ }^{2}$ Department of Neurosurgery, Spine and Spinal Cord Research Institute, Yonsei University College of Medicine, Seoul, \\ Republic of Korea \\ ${ }^{3}$ Department of Neurosurgery, Bundang Jesaeng General Hospital, Seongnam, Republic of Korea \\ ${ }^{4}$ Department of Neurosurgery, Cham Teun Teun Hospital, Guri, Republic of Korea \\ Correspondence should be addressed to Dong Ah Shin; shindongah@me.com
}

Received 1 December 2017; Accepted 27 February 2018; Published 1 April 2018

Academic Editor: Jacob Ablin

Copyright (c) 2018 Sang-Hyuk Park et al. This is an open access article distributed under the Creative Commons Attribution License, which permits unrestricted use, distribution, and reproduction in any medium, provided the original work is properly cited.

\begin{abstract}
Background. The correlation between epidurography contrast patterns and the clinical outcomes of percutaneous epidural neuroplasty (PEN) remains unclear. Objective. To analyze the correlation between postadhesiolysis epidurography contrast patterns and the clinical outcomes of patients who undergo lumbar PEN. Design. This study is a retrospective analysis of 78 consecutive patients who underwent lumbar PEN between April 2012 and March 2013. Setting. The analysis was done in the university hospital center. Method. The clinical outcomes of all patients were assessed before and 1, 3, 6, and 12 months after undergoing lumbar PEN. Specifically, the intensity of back and leg pain, quality of life, and procedural outcomes were evaluated using a visual analog scale (VAS), the Oswestry Disability Index (ODI), and the 12-Item Short-Form Health Survey (SF-12). Results. The VAS scores for back and leg pain, ODI score, and SF-12 score exhibited a significant improvement during the followup period $(P<0.01$ versus preprocedural scores). At most follow-up time points, patients exhibiting extraforaminal contrast distribution $(n=22)$ on postadhesiolysis epidurograms exhibited a similar improvement in VAS scores and a significantly better improvement in ODI and SF-12 scores compared with patients exhibiting intracanal contrast distribution $(n=56)$. Conclusion. Extraforaminal contrast distribution during lumbar PEN may be associated with better functional outcomes.
\end{abstract}

\section{Introduction}

Chronic low back pain is a common health problem with widespread socioeconomical reverberations which is experienced by most individuals at some point in their lives [1-3]. Several studies on low back pain have been conducted, and many treatments have been applied, with most physicians focusing on lumbar disc herniation as a leading cause for spinal interventions [4-7]. The demand for spinal interventions is increasing because of their minimal invasiveness and therapeutic efficacy [4-7]. Percutaneous epidural neuroplasty (PEN) is a novel, widely used technique for the lysis of microscopic adhesions surrounding nerve tissues and delivery of therapeutic drugs directly to the target area [8-15]. However, spinal interventions may not always be successful because of several barriers. For example, postoperative scarring inhibits epidural contrast spread, while foraminal stenosis blocks the extraforaminal extension of injected drugs $[16,17]$. In addition to obvious masses and canal narrowing, microscopic adhesions, which may be a result of chronic inflammation, can interrupt the spread of drugs. Although numerous studies have described PEN procedures, only a few have reported intraoperative findings that indicate the surgical outcomes [11, 13, 14, 17-19]. Epidurography is always performed during PEN procedures, but there is insufficient evidence supporting the usefulness of this procedure. Moreover, the 
correlation between epidurography contrast patterns and the clinical outcomes of PEN has not been frequently reported $[19,20]$. Postadhesiolysis epidurography patterns are assumed to be indicators of surgical outcomes. In addition, the presence of poor contrast spread on postadhesiolysis epidurograms may be associated with the requirement for repeat adhesiolysis procedures or a change in the target area for achieving better contrast patterns. In the present study, we assessed the clinical outcomes of lumbar PEN on the basis of contrast patterns on postadhesiolysis epidurograms.

\section{Methods}

2.1. Patient Selection. This retrospective analysis of medical and radiographic records included 78 patients who underwent lumbar PEN procedures between April 2012 and March 2013 at a single university hospital. The study protocol was approved by the ethics committee, and informed consent was obtained from all subjects. The inclusion criterion was chronic low back pain with or without leg pain due to lumbar disc herniation and/or lumbar spinal stenosis. All patients reported a history of discogenic or radicular symptoms refractory to conservative treatments for a minimum of 6 weeks. Diagnoses were established using magnetic resonance imaging (MRI) and/or computed tomography (CT) performed before PEN. Patients with a history of spinal surgery and those with cauda equina syndrome, bleeding diathesis, associated somatic or psychiatric disease, vertebral fractures, pregnancy, and tumors or other underlying systemic diseases that could significantly affect the procedural outcomes were excluded. All procedures were performed by one of the authors (DAS) using the same procedural protocol.

2.2. PEN Procedure. A standard PEN procedure was used to lyse adhesions and achieve nerve blockades in all patients, as previously described $[11,14]$. A 1-day protocol was followed. The patient was placed on a radiolucent table in the prone position, and the procedure was performed under fluoroscopic guidance. The coccygeal and sacral regions were disinfected with $10 \%$ Betadine, and the surgical site was draped in the usual aseptic manner. The sacral hiatus was anesthetized with $1 \%$ xylocaine. Then, a $20-\mathrm{G}$ Tuohy needle was introduced into the epidural space below the level of S3. A total of $3 \mathrm{~mL}$ of the contrast agent (Omnipaque, GE Healthcare Korea, Seoul, Republic of Korea) was instilled to confirm the epidural space and preadhesiolysis status. Both anteroposterior and lateral fluoroscopic views were obtained. We also assessed the development of any adverse reactions. On confirmation of the target for PEN, a catheter specialized for adhesiolysis (TUN-L-KATH; Epimed, TX, United States) was gently inserted toward the target site. For better catheter manipulation, the tip was bent for an approximate length of $1 \mathrm{~cm}$. The catheter was easily navigated by rotation. Once it reached the target site, a second epidurogram was obtained by injecting $3 \mathrm{~mL}$ of the contrast agent for the identification of filling defects or cutoff signs surrounding the target area. When the tip of the catheter touched the target site or the contrast agent exerted pressure on the lesion, patients were asked to report provoked symptoms. According to the surgical records, they frequently reported pain similar to what they had been suffering. Both mechanical and chemical adhesiolysis were performed. The former was achieved through pushing, pulling, and rotating movements of the catheter, while the latter was achieved by the injection of a mixture comprising $0.9 \%$ normal saline $(10 \mathrm{~mL})$ and $3000 \mathrm{U}$ of hyaluronidase (H-lase; $1500 \mathrm{U} / \mathrm{mL}$; Kuhnil, South Korea). Following adhesiolysis, a third epidurogram was obtained using $3 \mathrm{~mL}$ of the contrast agent. The patterns of contrast dispersal were examined on both anteroposterior and lateral fluoroscopic views. All epidurogram images were saved in the Digital Imaging and Communications in Medicine format for future analysis, and the third epidurogram was used for analysis in our study. Finally, a mixture of $0.2 \%$ ropivacaine (8 mL; Naropin; Astrazeneca Korea, Seoul, Republic of Korea) and $40 \%$ triamcinolone acetonide $(20 \mathrm{mg}$; triamcinolone; DongKwang, Seoul, Republic of Korea) was slowly injected. The stored movie clips and still images were independently reviewed twice by two authors who were blinded to the patient data (SHP and PGC).

2.3. Epidurography Contrast Patterns. Postadhesiolysis epidurography contrast patterns were defined and classified into five grades according to the system proposed by Mathis et al. [21]: grade 1, contrast spread to the medial or midline zone of the ipsilateral or contralateral epidural space; grade 2, contrast spread to the lateral epidural space, proximal to the medial border of the neural foramen; grade 3, contrast spread to the intraforaminal space, not extending to the lateral border of the neural foramen; grade 4, contrast spread to the intraforaminal space, extending to but not crossing the lateral border of the neural foramen; and grade 5, contrast spread beyond the lateral border of the neural foramen (Figure 1). For the comparison of clinical outcomes according to the contrast distribution pattern on epidurograms, patients with grade 1 , grade 2 , and grade 3 patterns were assigned to a group exhibiting limited intracanal spread (IC group), while patients with grade 4 and grade 5 patterns were assigned to a group exhibiting extended extraforaminal spread (EF group).

\subsection{Follow-Up and Assessment of Surgical Outcomes. All} patients were clinically evaluated before and 1, 3, 6, and 12 months after PEN by a nurse specialized in pain management and blinded to the treatment details. The intensity of leg and back pain was assessed using a subjective visual analog scale (VAS) calibrated from 0 to $10(0=$ no pain and $10=$ the worst pain imaginable). For functional assessments, the Korean versions of the Oswestry Disability Index (ODI) and 12-Item Short-Form Health Survey (SF-12) were used $[22,23]$. ODI evaluated the clinical effectiveness of PEN in terms of pain reduction and functional improvement, with the score ranging from 0 to 50 . SF-12 incorporates two dimensions: a physical component summary (PCS) and a mental component summary (MCS). PCS and MCS scores are computed from the scores for 12 questions and range from 0 to 100 , with a higher score representing better physical or mental health [19]. 

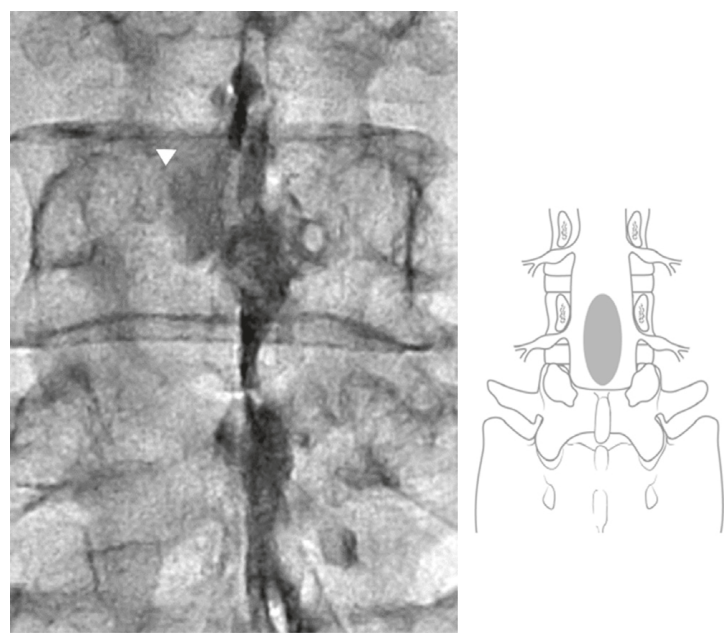

(a)

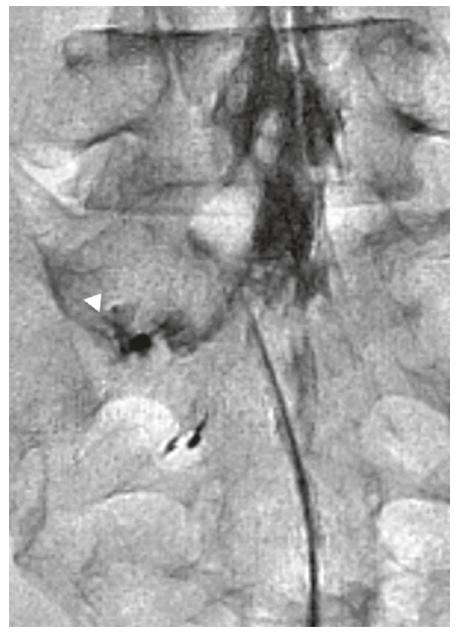

(c)
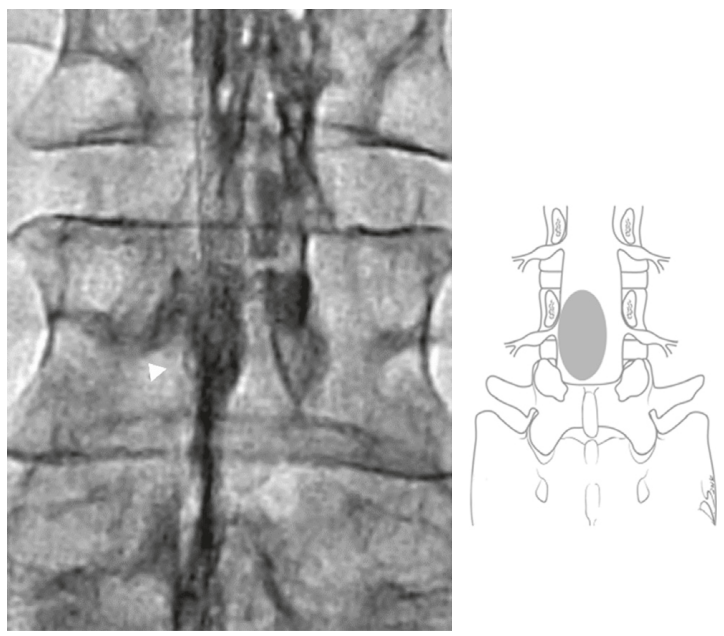

(b)
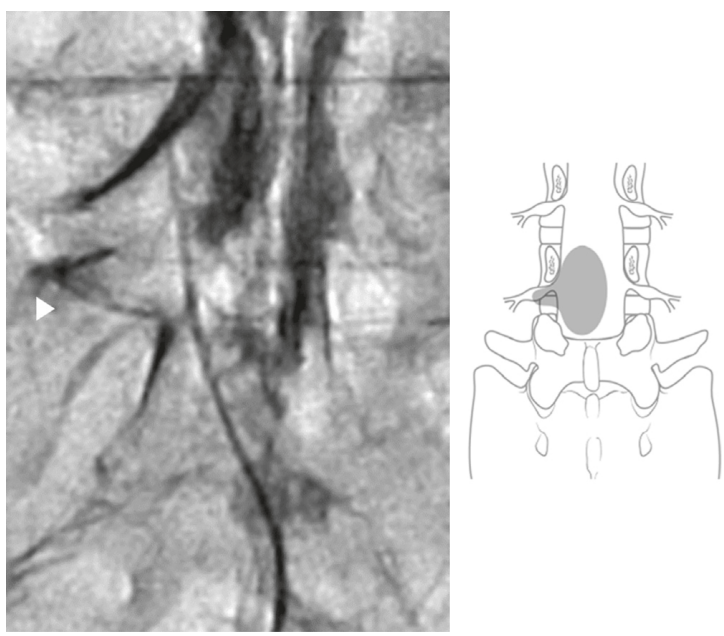

(d)
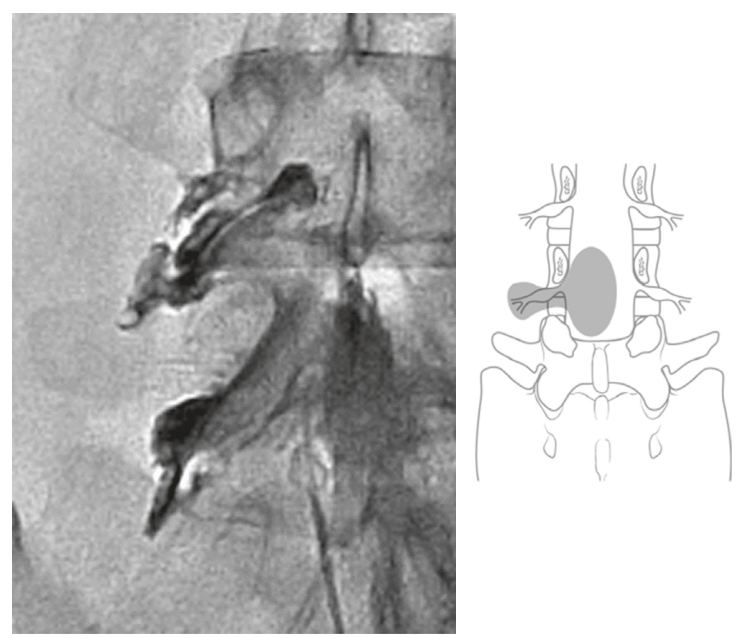

(e)

Figure 1: Modified classification of epidurography contrast patterns. (a) Grade 1: contrast spread to the medial or midline zone of the ipsilateral or contralateral epidural space, (b) grade 2: contrast spread to the lateral epidural space, proximal to the medial border of the neural foramen, (c) grade 3: contrast spread to the intraforaminal space, not extending to the lateral border of the neural foramen, (d) grade 4: contrast spread to the intraforaminal space, extending to but not crossing the lateral border of the neural foramen, and (e) grade 5: contrast spread beyond the lateral border of the neural foramen. Patients with grade 1, grade 2 , and grade 3 patterns were assigned to a group exhibiting limited intracanal spread (IC group), while patients with grade 4 and grade 5 patterns were assigned to a group exhibiting extended extraforaminal spread (EF group). 
2.5. Statistical Analysis. Age, the duration of symptoms and follow-up, and VAS, ODI, and SF-12 scores are expressed as means \pm standard deviations. Student's $t$-tests were used to assess differences in VAS, ODI, and SF-12 scores at each time point between the EF and IC groups. Chi-square tests were used to compare clinical outcomes between the two groups. Spearman's rank correlation tests were used to determine the correlation between epidurography contrast patterns and clinical outcomes. All statistical analyses were performed using the Statistical Package for the Social Sciences software (SPSS Inc., Chicago, Illinois, USA). A $P$ value of $<0.05$ was considered statistically significant.

\section{Results}

3.1. Patient Characteristics. A total of 78 patients $(27$ men and 51 women) were included in this study. All patients were clinically followed up for more than 11 months. The mean age was $58.1 \pm 14.1$ years (range, 18 to 84 years), and the average duration of major symptoms before PEN was $7.1 \pm 4.1$ months (range, 2 to 31 months). Before PEN, the VAS score for back pain, VAS score for leg pain, ODI score, and SF-12 score were $5.8 \pm 2.6, \quad 5.0 \pm 3.1, \quad 19.4 \pm 7.0$, and $30.0 \pm 7.2$, respectively (Table 1).

3.2. Treatment Outcomes. The clinical outcomes of patients exhibited a significant improvement during the follow-up period. At 1, 3, 6, and 12 months after PEN, the VAS score for back pain was $2.6 \pm 2.3,3.1 \pm 2.1,3.0 \pm 2.0$, and $3.4 \pm 2.4$, respectively; the VAS score for leg pain was $1.9 \pm 2.0,2.6 \pm$ $2.1,2.6 \pm 2.2$, and $2.7 \pm 2.6$, respectively; the ODI score was $10.6 \pm 8.0,11.7 \pm 7.4,11.0 \pm 7.3$, and $11.0 \pm 8.0$, respectively; and the SF-12 score was $41.8 \pm 9.6,40.5 \pm 9.0,41.2 \pm 9.2$, and $41.2 \pm 10.2$, respectively $(P<0.01$ when compared with preprocedural scores; Figure 2).

\subsection{Postadhesiolysis Epidurography Contrast Patterns and} Clinical Outcomes. The patients were categorized into IC and EF groups according to the contrast patterns on postadhesiolysis epidurograms. In the IC group $(n=56)$, there were 19,25 , and 12 patients with grade 1 , grade 2 , and grade 3 patterns, respectively. In the EF group $(n=22)$, there were 7 and 15 patients with grade 4 and grade 5 patterns, respectively. Before PEN, the VAS score for back pain was $5.7 \pm 2.6$ and $6.0 \pm 2.4(P=0.639)$, the VAS score for leg pain was $4.7 \pm 3.1$ and $6.0 \pm 2.8(P=0.101)$, the ODI score was $20.0 \pm 6.9$ and $17.8 \pm 7.1(P=0.222)$, and the SF12 score was $29.6 \pm 7.0$ and $31.0 \pm 7.7(P=0.452)$ in the IC and EF groups, respectively, with no significant differences between groups. At most follow-up time points, the EF group exhibited a similar improvement in VAS scores and a significantly better improvement in ODI and SF-12 scores compared with the IC group (Table 2). Thus, extraforaminal contrast spread was associated with a tendency for decreased pain and significantly better quality of life (Figure 3; Spearman's rank correlation test $(P<0.05))$. A representative case of a patient with radicular pain in the left leg who
TABLE 1: Baseline characteristics of patients with chronic low back pain with or without leg pain who underwent lumbar percutaneous epidural neuroplasty.

\begin{tabular}{|c|c|c|}
\hline & Variable & Range \\
\hline $\begin{array}{l}\text { Number of patients (men: } \\
\text { women) }\end{array}$ & 78 & $27: 51$ \\
\hline Age (years) & $58.1 \pm 14.1$ & $18-84$ \\
\hline Symptom duration (months) & $7.1 \pm 4.1$ & $2-31$ \\
\hline Follow-up duration (months) & $12.7 \pm 1.0$ & $11-16$ \\
\hline \multirow{3}{*}{$\begin{array}{ll}\text { VAS score } & \begin{array}{l}\text { Back pain } \\
\text { Leg pain }\end{array} \\
\text { ODI score } & \end{array}$} & $5.8 \pm 2.6$ & - \\
\hline & $5.0 \pm 3.1$ & - \\
\hline & $19.4 \pm 7.0$ & - \\
\hline SF-12 score & $30.0 \pm 7.2$ & - \\
\hline
\end{tabular}

VAS: visual analog scale; ODI: Oswestry Disability Index; SF-12: 12-Item Short-Form Health Survey.

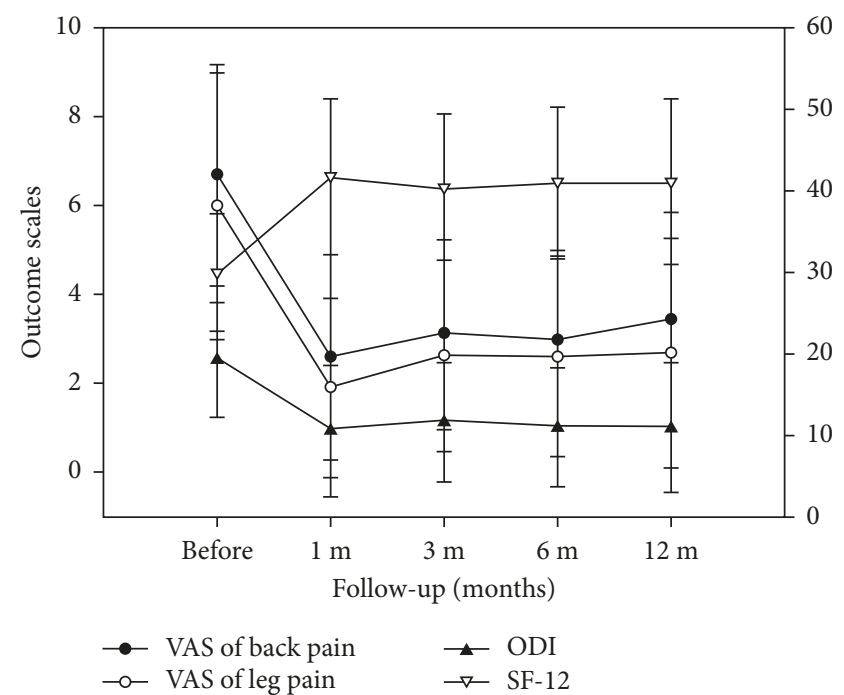

Figure 2: Clinical outcomes of patients who underwent lumbar PEN. VAS, ODI, and SF-12 scores before and 1, 3, 6, and 12 months after lumbar PEN. All postprocedural clinical scores have significantly improved compared with preprocedural scores $(P<0.001)$. VAS: visual analog scale, ODI: Oswestry Disability Index, SF-12: 12-Item Short-Form Health Survey, and PEN: percutaneous epidural neuroplasty.

exhibited significant pain relief and functional recovery after $\mathrm{PEN}$ is depicted in Figure 4.

\section{Discussion}

Epidurograms provide anatomical information and help in confirming accurate needle placement. Spinal interventions can be safely performed using a fluoroscope and epidurograms with radiopaque dye. The aim of the present study was to evaluate the correlation between contrast patterns on postadhesiolysis epidurograms and clinical outcomes in patients who underwent lumbar PEN. We observed that PEN ameliorated the pain associated with lumbar disc herniation and/or lumbar spinal stenosis. VAS scores for leg and back pain, ODI scores, and SF-12 scores exhibited a significant improvement over the entire 12-month follow-up period. Racz et al. [24] reported similar results in their study, where 
TABLE 2: Clinical outcomes of patients with limited intracanal contrast spread (IC group) and those with extended extraforaminal contrast spread (EC group) on postadhesiolysis epidurograms obtained during lumbar percutaneous epidural neuroplasty (PEN).

\begin{tabular}{|c|c|c|c|c|c|}
\hline & & VAS score for back pain & VAS score for leg pain & ODI score & SF-12 score \\
\hline \multirow{3}{*}{ Before PEN } & IC group & $5.7 \pm 2.6$ & $4.7 \pm 3.1$ & $20.0 \pm 6.9$ & $29.6 \pm 7.0$ \\
\hline & EF group & $6.0 \pm 2.4$ & $6.0 \pm 2.8$ & $17.8 \pm 7.1$ & $31.0 \pm 7.7$ \\
\hline & $P$ value & 0.639 & 0.101 & 0.222 & 0.452 \\
\hline \multirow{3}{*}{1 month after PEN } & IC group & $2.9 \pm 2.3$ & $2.0 \pm 2.1$ & $12.1 \pm 8.4$ & $40.3 \pm 10.2$ \\
\hline & EF group & $1.9 \pm 2.1$ & $1.7 \pm 1.8$ & $6.7 \pm 5.6$ & $45.7 \pm 8.5$ \\
\hline & $P$ value & 0.076 & 0.532 & 0.007 & 0.023 \\
\hline \multirow{3}{*}{3 months after PEN } & IC group & $3.3 \pm 2.2$ & $2.6 \pm 2.2$ & $13.0 \pm 7.6$ & $39.4 \pm 9.6$ \\
\hline & EF group & $2.7 \pm 2.1$ & $2.6 \pm 1.9$ & $8.5 \pm 5.5$ & $43.4 \pm 8.7$ \\
\hline & $P$ value & 0.317 & 0.924 & 0.015 & 0.043 \\
\hline \multirow{3}{*}{6 months after PEN } & IC group & $3.3 \pm 2.0$ & $2.7 \pm 2.3$ & $12.6 \pm 7.6$ & $39.4 \pm 9.4$ \\
\hline & EF group & $2.2 \pm 2.0$ & $2.2 \pm 2.1$ & $7.1 \pm 4.8$ & $46.0 \pm 6.9$ \\
\hline & $P$ value & 0.032 & 0.372 & 0.002 & 0.007 \\
\hline \multirow{3}{*}{12 months after PEN } & IC group & $3.7 \pm 2.4$ & $2.9 \pm 2.5$ & $12.6 \pm 8.2$ & $39.3 \pm 10.3$ \\
\hline & EF group & $2.7 \pm 2.6$ & $2.2 \pm 2.7$ & $7.0 \pm 6.0$ & $46.1 \pm 8.3$ \\
\hline & $P$ value & 0.087 & 0.274 & 0.005 & 0.007 \\
\hline
\end{tabular}

VAS: visual analog scale; ODI: Oswestry Disability Index; SF-12: 12-Item Short-Form Health Survey.

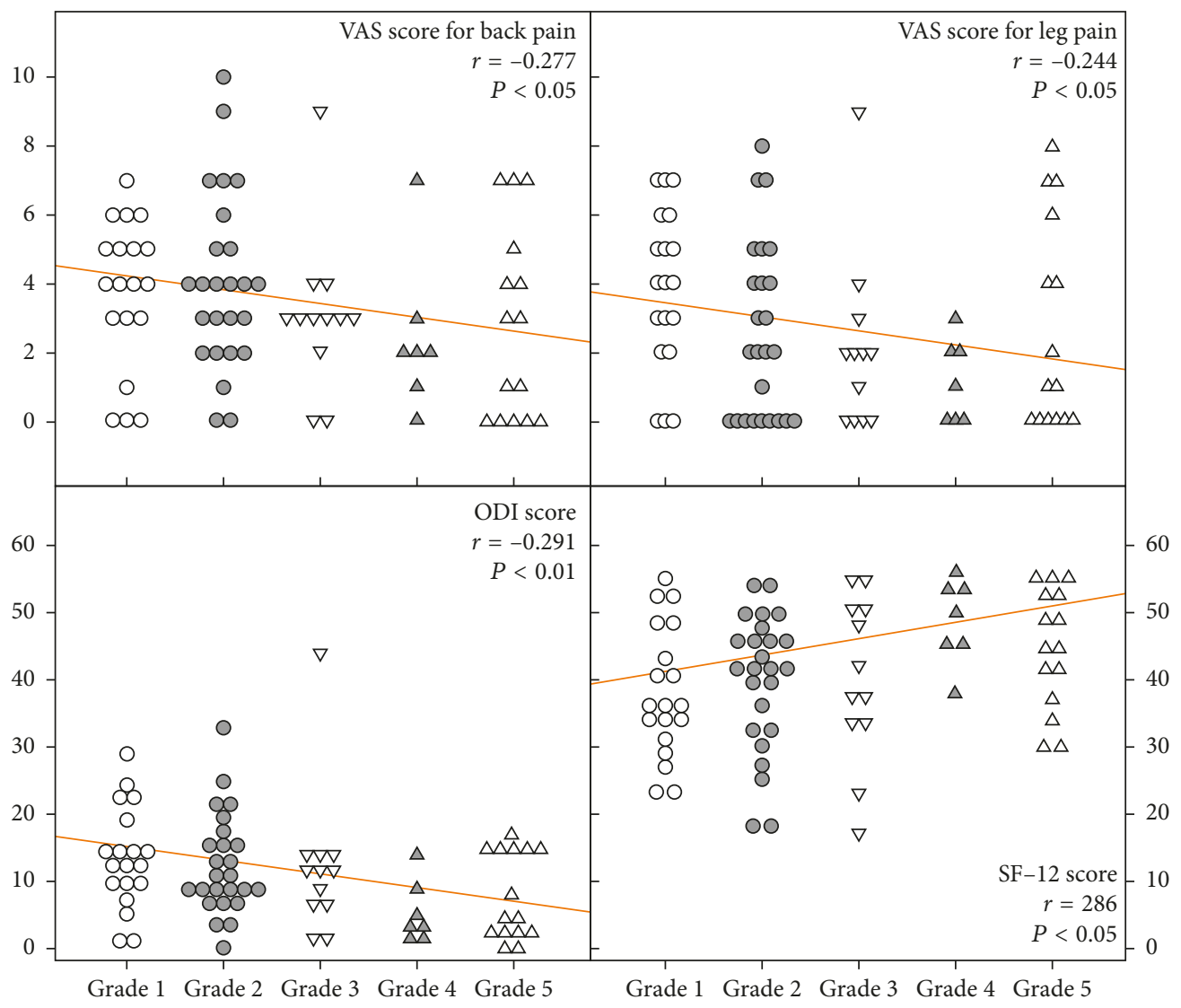

FIGURE 3: Correlation between epidurography contrast spread after adhesiolysis during PEN and clinical outcomes. Extraforaminal contrast spread (grades 4 and 5) is associated with a tendency for decreased pain and significantly better quality of life compared with intracanal spread (grades 1, 2, and 3). PEN: percutaneous epidural neuroplasty, VAS: visual analog scale, ODI: Oswestry Disability Index, and SF-12: 12-Item Short-Form Health Survey.

immediate pain relief was observed after PEN in more than $70 \%$ patients and the outcomes were maintained for 6 months. The success rate for PEN is reported to be $50 \%-71 \%$ $[11,14,18,25,26]$. The present study also showed good clinical outcomes, with the greatest improvement observed at
1 month after PEN. Moreover, the improved outcomes were maintained without much variation for 1 year after the procedure. The immediate effect of PEN is believed to result from the combined effects of mechanical adhesiolysis, chemical adhesiolysis, and local lavage of inflammatory 


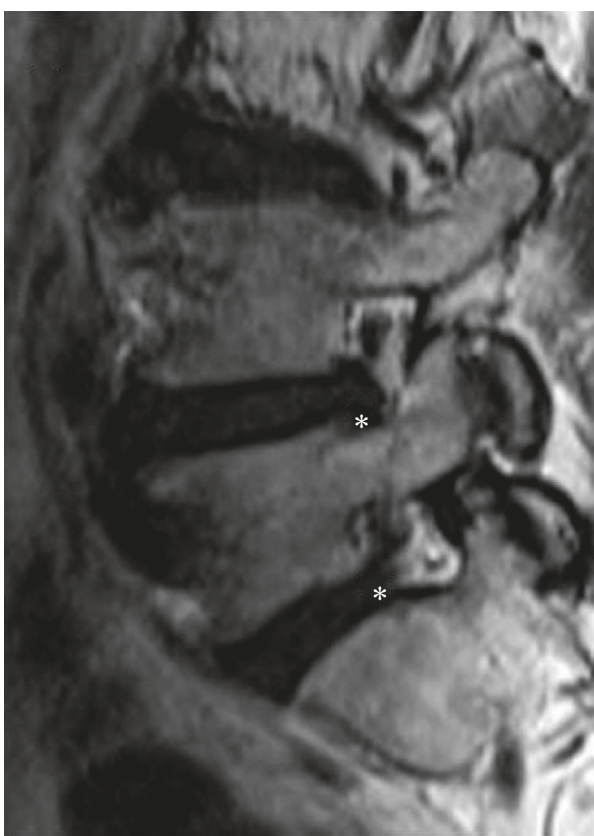

(a)

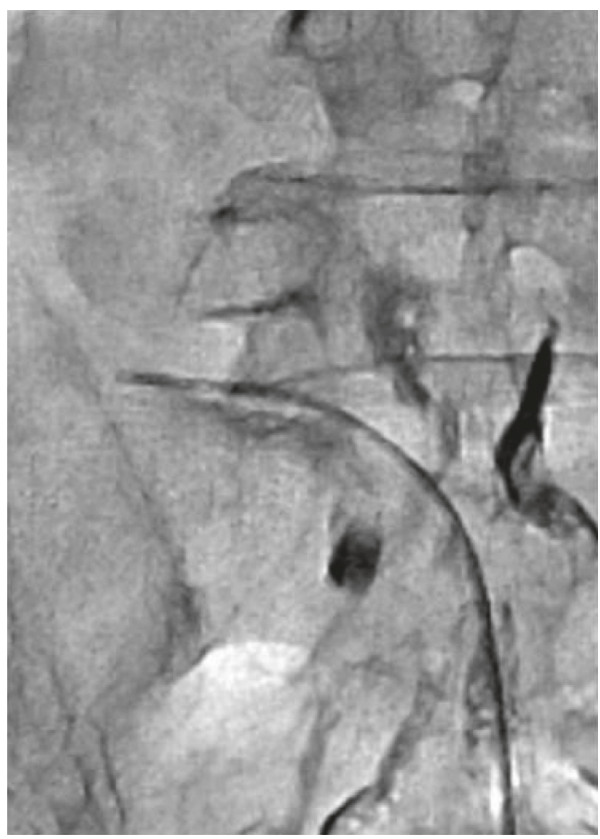

(c)

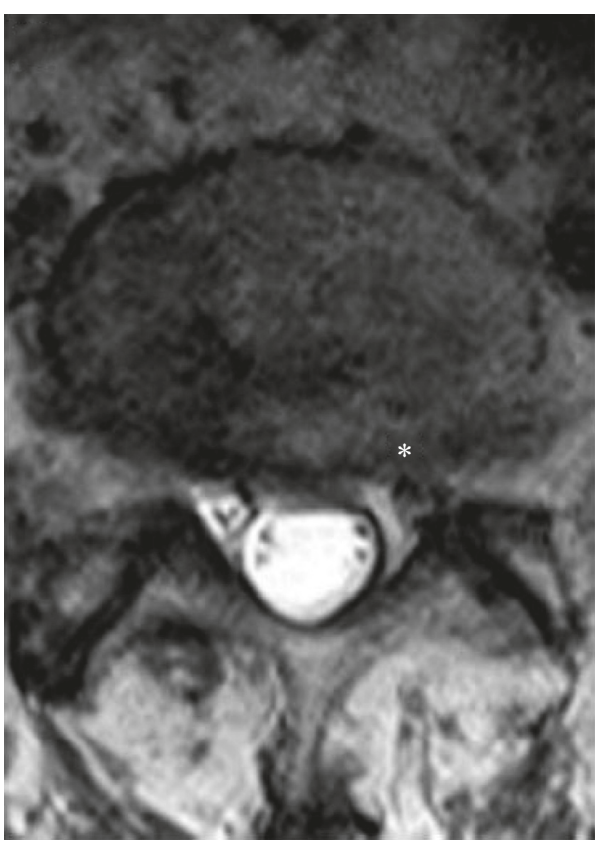

(b)

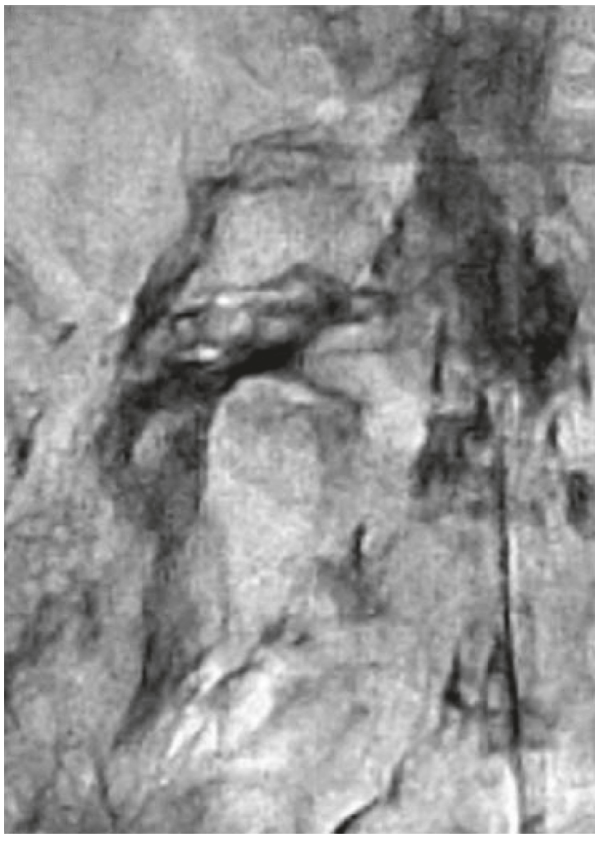

(d)

Figure 4: Representative case of a 60-year-old man with radicular pain in the left leg who underwent lumbar PEN. (a, b) Magnetic resonance imaging shows lumbar disc herniation and foraminal stenosis at the level of L4-5-S1 (asterisks). (c) The first epidurogram shows a filling defect at the left L5-S1 foramen. The catheter is inserted to the stenotic foramen, and mechanical adhesiolysis is attempted. (d) The final postadhesiolysis epidurogram shows excellent extraforaminal contrast spread. The patient exhibited significant pain relief and functional recovery after PEN. PEN: percutaneous epidural neuroplasty.

mediators, which induce desensitization, neuromodulation, and local anesthesia [8, 27].

In the present study, we found that the clinical outcomes of lumbar PEN were correlated with the presence of extraforaminal contrast spread on the final epidurogram obtained after adhesiolysis. Extraforaminal contrast spread is believed to be an indicator of successful adhesiolysis. The purpose of
PEN is to eliminate the barriers in the epidural space that disturb drug delivery. Extraforaminal contrast spread may be related to the creation of channels around the target site through adhesiolysis, and it facilitates drug delivery to the lesion site. Exposure to the ideal amount of medication can result in better pain reduction. It can also enhance the recovery of the perineural circulation, which can improve 
neural congestion and decrease the associated pain. The results of our study confirmed that access to the exact position of the lesion site is associated with a better outcome. These findings suggest that mechanical adhesiolysis is meaningful only at the lesion site. Moreover, if the exact lesion site cannot be reached, both adhesiolysis and drug delivery will fail and the target area will remain untreated.

In 1991, Kuslich et al. [28] demonstrated pain-sensitive structures in the spinal canal using mechanical and electrical stimulation. These structures include the annulus, nerve roots, posterior longitudinal ligament, facet joints, tendons, ligaments, and fascia [29]. There is evidence suggesting that the distribution of pain generators is increased in the ventral lateral space $[29,30]$. Accordingly, we expected that placement of the catheter in the ventral epidural space would be strongly correlated with better outcomes. However, we could not confirm a direct correlation between ventral placement of the catheter and improved outcomes. Instead, we observed that ventral placement of the catheter tip was correlated more with the possibility of extraforaminal contrast spread, which resulted in better outcomes. It is assumed that adhesiolysis, rather than ventral catheter placement, affects clinical outcomes. Further studies with larger patient samples are necessary to clarify the direct effects of ventral catheter placement. We also found that the epidurography contrast pattern showed no significant differences among the five grades. Therefore, we suggest that division of the pattern into two grades may be adequate for evaluating the surgical outcomes of PEN.

In the present study, there were no direct correlations between cutoff signs or filling defects and the clinical outcomes of PEN. In our practice, we cannot easily resolve cutoff signs and filling defects. Cutoff signs are a result of severe spinal stenosis, while filling defects are frequently associated with disc herniations. Currently, gross adhesions cannot be easily lysed by soft PEN. However, regardless of the presence of cutoff signs or filling defects, better outcomes were achieved with extraforaminal contrast spread. There may be microscopic adhesions that are more relevant and can be lysed by soft PEN. Park et al. also reported that there was no correlation between the degree of pain relief and epidural filling defects in patients with lumbar stenosis [31].

This study has several limitations. These include the small sample size, the unequal groups, the short follow-up duration, and the retrospective design. Future studies should ideally include an appropriate control group. Nevertheless, the strength of our study is the use of clinical outcome measures, namely, ODI and SF-12, for assessment of the quality of life in addition to pain scores. Furthermore, the results of the study are more in line with the prognosis because the patients received a single treatment rather than repetitive treatments. With the use of epidurograms, physicians can design and plan adhesiolysis that aims at a precise target point and creates effective tunnels, which will help in predicting the prognosis.

\section{Conclusions}

In conclusion, the findings of our study suggest that the contrast patterns on final epidurograms obtained after adhesiolysis during PEN are indicators of clinical outcomes.
Specifically, extraforaminal contrast spread during PEN is associated with better functional outcomes.

\section{Conflicts of Interest}

The authors declare that there are no conflicts of interest regarding the publication of this article.

\section{Authors' Contributions}

Sang-Hyuk Park and Gyu Yeul Ji contributed equally to this study.

\section{Acknowledgments}

This study was supported by a grant from the Korea Evaluation Institute of Industrial Technology (10043086) and a grant from the Korea Health Technology R\&D Project through the Korea Health Industry Development Institute (KHIDI), funded by the Ministry of Health and Welfare, Republic of Korea (HC15C1320). The authors would like to thank Editage (www.editage.com) for English language editing and publication support.

\section{References}

[1] A. Sharan, J. Riley, and C. Hoelscher, "An overview of chronic spinal pain: revisiting diagnostic categories and exploring an evolving role for neurostimulation," Spine, vol. 42, no. 14, pp. S35-S40, 2017.

[2] R. A. Deyo, S. K. Mirza, and B. I. Martin, "Back pain prevalence and visit rates: estimates from U.S. national surveys, 2002," Spine, vol. 31, no. 23, pp. 2724-2727, 2006.

[3] D. Hoy, P. Brooks, F. Blyth, and R. Buchbinder, "The epidemiology of low back pain," Best Practice and Research Clinical Rheumatology, vol. 24, no. 6, pp. 769-781, 2010.

[4] L. Manchikanti, S. Datta, R. Derby et al., "A critical review of the American Pain Society clinical practice guidelines for interventional techniques: part 1. Diagnostic interventions," Pain Physician, vol. 13, no. 3, pp. E141-E174, 2010.

[5] L. Manchikanti, S. Abdi, S. Atluri et al., "An update of comprehensive evidence-based guidelines for interventional techniques in chronic spinal pain. Part II: guidance and recommendations," Pain Physician, vol. 16, no. 2, pp. S49S283, 2013.

[6] M. V. Boswell, A. M. Trescot, S. Datta et al., "Interventional techniques: evidence-based practice guidelines in the management of chronic spinal pain," Pain Physician, vol. 10, no. 1, pp. 7-111, 2007.

[7] P. J. Orrock and S. P. Myers, "Osteopathic intervention in chronic non-specific low back pain: a systematic review," BMC Musculoskeletal Disorders, vol. 14, no. 1, p. 129, 2013.

[8] L. Gerdesmeyer, S. Wagenpfeil, C. Birkenmaier et al., "Percutaneous epidural lysis of adhesions in chronic lumbar radicular pain: a randomized, double-blind, placebo-controlled trial," Pain Physician, vol. 16, no. 3, pp. 185-196, 2013.

[9] L. Manchikanti, V. Singh, K. A. Cash, and V. Pampati, "Assessment of effectiveness of percutaneous adhesiolysis and caudal epidural injections in managing post lumbar surgery syndrome: 2-year follow-up of a randomized, controlled trial," Journal of Pain Research, vol. 5, pp. 597-608, 2012. 
[10] L. Manchikanti and C. E. Bakhit, "Percutaneous lysis of epidural adhesions," Pain Physician, vol. 3, no. 1, pp. 46-64, 2000.

[11] G. Y. Ji, C. H. Oh, B. Moon et al., "Efficacy of percutaneous epidural neuroplasty does not correlate with dural sac crosssectional area in single level disc disease," Yonsei Medical Journal, vol. 56, no. 3, pp. 691-697, 2015.

[12] G. Y. Ji, C. H. Oh, K. S. Won et al., "Randomized controlled study of percutaneous epidural neuroplasty using Racz catheter and epidural steroid injection in cervical disc disease," Pain Physician, vol. 19, no. 2, pp. 39-48, 2016.

[13] S. H. Moon, J. I. Lee, H. S. Cho, J. W. Shin, and W. U. Koh, "Factors for predicting favorable outcome of percutaneous epidural adhesiolysis for lumbar disc herniation," Pain Research and Management, vol. 2017, Article ID 1494538, 10 pages, 2017.

[14] C. H. Oh, G. Y. Ji, P. G. Cho et al., "The catheter tip position and effects of percutaneous epidural neuroplasty in patients with lumbar disc disease during 6-months of follow-up," Pain Physician, vol. 17, no. 5, pp. E599-E608, 2014.

[15] G. B. Racz, J. E. Heavner, and A. Trescot, "Percutaneous lysis of epidural adhesions-evidence for safety and efficacy," Pain Practice, vol. 8, no. 4, pp. 277-286, 2008.

[16] N. Takeshima, H. Miyakawa, K. Okuda et al., "Evaluation of the therapeutic results of epiduroscopic adhesiolysis for failed back surgery syndrome," British Journal of Anaesthesia, vol. 102, no. 3, pp. 400-407, 2009.

[17] R. F. McLain, L. Kapural, and N. A. Mekhail, "Epidural steroid therapy for back and leg pain: mechanisms of action and efficacy," Spine Journal, vol. 5, no. 2, pp. 191-201, 2005.

[18] C. H. Park, S. H. Lee, and J. Y. Jung, "Dural sac cross-sectional area does not correlate with efficacy of percutaneous adhesiolysis in single level lumbar spinal stenosis," Pain Physician, vol. 14, no. 4, pp. 377-382, 2011.

[19] S. H. Kim, M. W. Jo, J. Ahn, M. Ock, S. Shin, and J. Park, "Assessment of psychometric properties of the Korean SF-12 v2 in the general population," BMC Public Health, vol. 14, p. 1086, 2014.

[20] Y. J. Han, M. N. Lee, M. J. Cho, H. J. Park, D. E. Moon, and Y. H. Kim, "Contrast runoff correlates with the clinical outcome of cervical epidural neuroplasty using a Racz catheter," Pain Physician, vol. 19, no. 7, pp. E1035-E1040, 2016.

[21] J. M. Mathis, S. Golovac, and C. H. Cho, "Pharmaceuticals used in image-guided spine interventions," Neuroimaging Clinics of North America, vol. 20, no. 2, pp. 215-222, 2010.

[22] J. C. Fairbank and P. B. Pynsent, "The Oswestry Disability Index," Spine, vol. 25, no. 22, pp. 2940-2952, 2000.

[23] D. Y. Kim, S. H. Lee, H. Y. Lee et al., "Validation of the Korean version of the Oswestry disability index," Spine, vol. 30, no. 5, pp. E123-E127, 2005.

[24] G. B. Racz, J. E. Heavner, and P. Raj, "Percutaneous epidural neuroplasty: prospective one year follow-up," Pain Digest, vol. 9, pp. 97-102, 1999.

[25] L. Manchikanti, K. A. Cash, C. D. McManus, V. Pampati, V. Singh, and R. Benyamin, "The preliminary results of a comparative effectiveness evaluation of adhesiolysis and caudal epidural injections in managing chronic low back pain secondary to spinal stenosis: a randomized, equivalence controlled trial," Pain Physician, vol. 12, no. 6, pp. E341-E354, 2009.

[26] L. Manchikanti, K. A. Cash, C. D. McManus, and V. Pampati, "Assessment of effectiveness of percutaneous adhesiolysis in managing chronic low back pain secondary to lumbar central spinal canal stenosis," International Journal of Medical Sciences, vol. 10, no. 1, pp. 50-59, 2013.

[27] C. Birkenmaier, S. Baumert, C. Schroeder, V. Jansson, and B. Wegener, "A biomechanical evaluation of the epidural neurolysis procedure," Pain Physician, vol. 15, no. 1, pp. E89-E97, 2012.

[28] S. D. Kuslich, C. L. Ulstrom, and C. J. Michael, "The tissue origin of low back pain and sciatica: a report of pain response to tissue stimulation during operations on the lumbar spine using local anesthesia," Orthopedic Clinics of North America, vol. 22, no. 2, pp. 181-187, 1991.

[29] S. Nakamura, K. Takahashi, Y. Takahashi, T. Morinaga, Y. Shimada, and H. Moriya, "Origin of nerves supplying the posterior portion of lumbar intervertebral discs in rats," Spine, vol. 21, no. 8, pp. 917-924, 1996.

[30] A. Veihelmann, C. Devens, H. Trouillier, C. Birkenmaier, L. Gerdesmeyer, and H. J. Refior, "Epidural neuroplasty versus physiotherapy to relieve pain in patients with sciatica: a prospective randomized blinded clinical trial," Journal of Orthopaedic Science, vol. 11, no. 4, pp. 365-369, 2006.

[31] C. Hong Park and S. Ho Lee, "Epidurographic findings following percutaneous epidural adhesiolysis failed to correlate with level of pain reduction in patients with lumbar spinal stenosis," Pain Medicine, vol. 18, no. 5, pp. 842-845, 2017. 


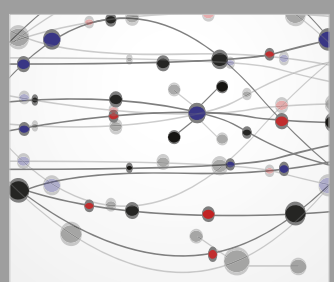

The Scientific World Journal
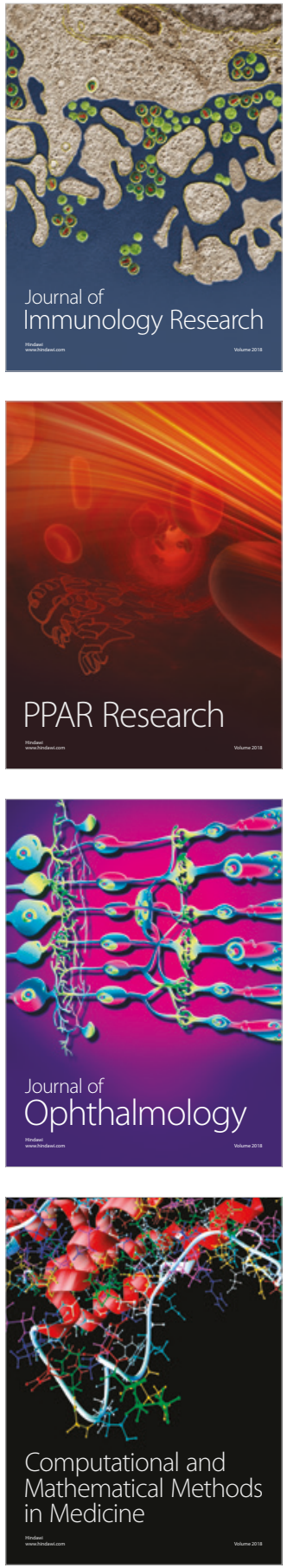



Gastroenterology Research and Practice

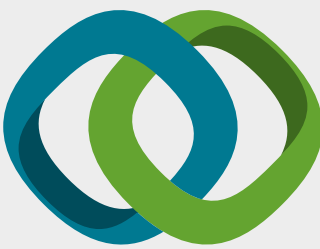

\section{Hindawi}

Submit your manuscripts at

www.hindawi.com
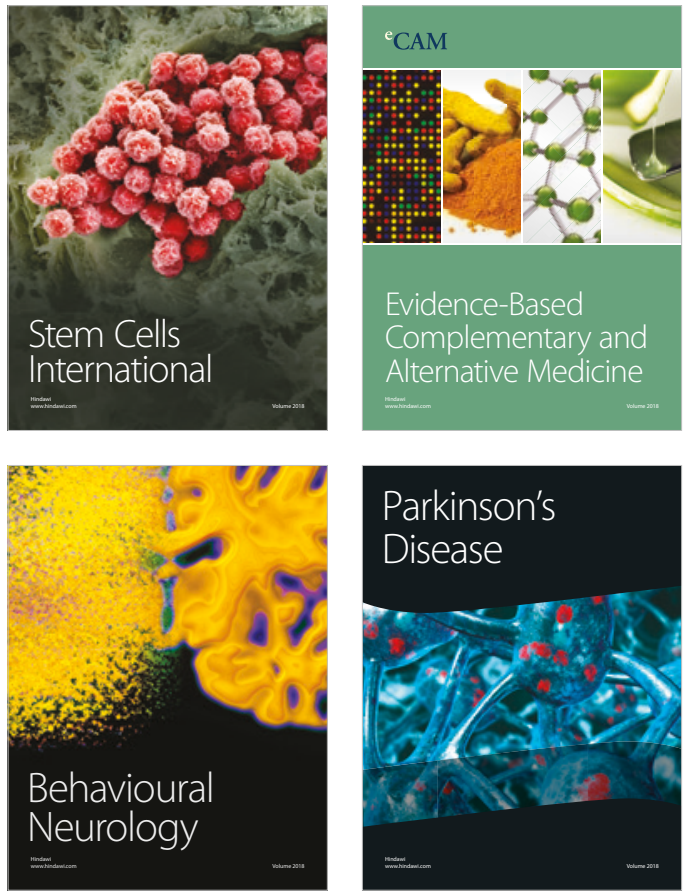

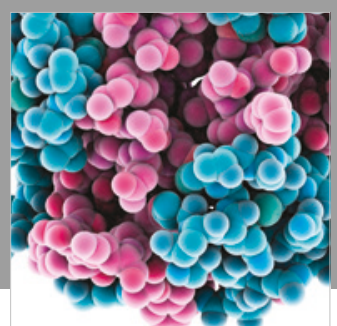

ournal of

Diabetes Research

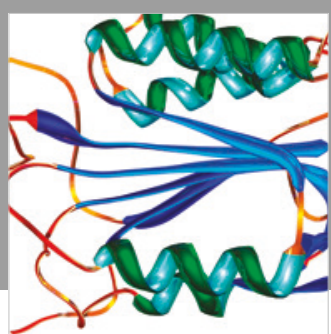

Disease Markers
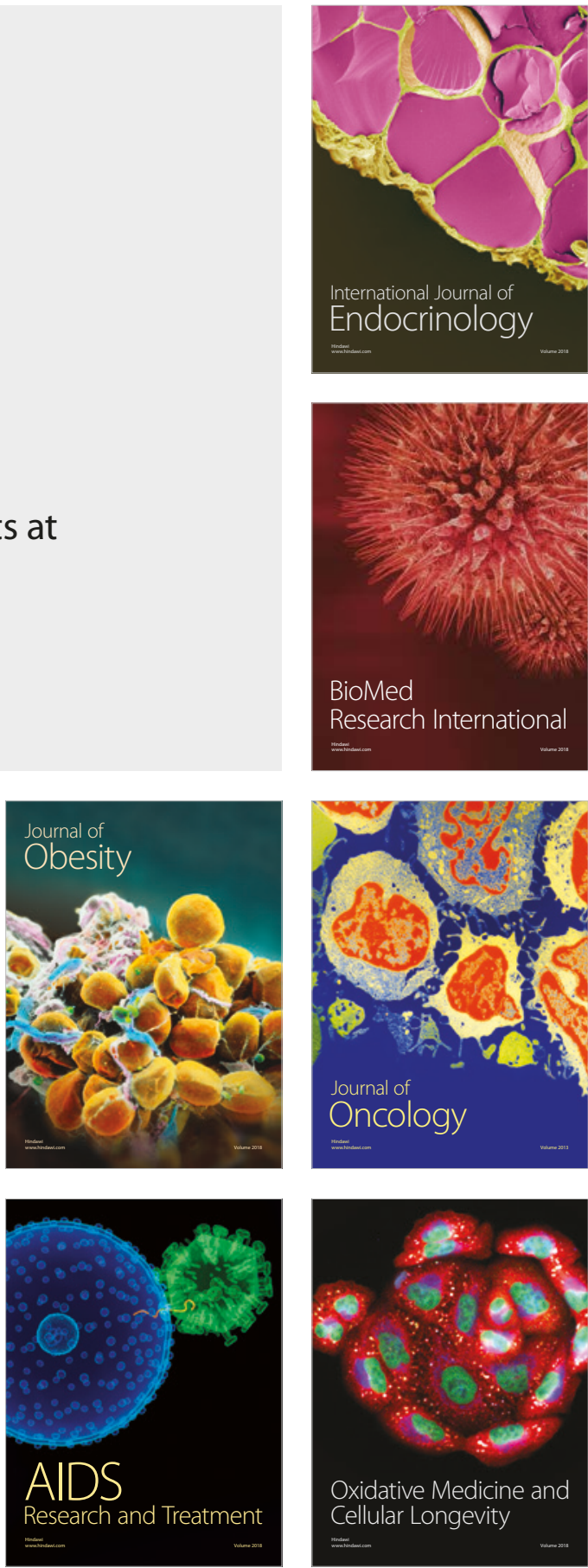